\title{
INFORMACIÓN PREVENTIVA SOBRE HIPERTENSIÓN ARTERIAL Y ESTILO DE VIDA, EN ADULTOS MAYORES DEL CENTRO DE SALUD LOS AQUIJES, ICA 2019
}

\author{
Preventive information on arterial hypertension and lifestyle in older adults from the Aquijes \\ Health Center, Ica 2019
}

Fátima Gregoria Huamán Vega ${ }^{1, a}$

${ }^{1}$ Facultad de Enfermería. Universidad Nacional San Luis Gonzaga. Ica, Perú.

a Licenciada en Enfermería

\section{RESUMEN}

El estudio tuvo por Objetivo: Determinar la relación entre la información preventiva sobre hipertensión arterial y el estilo de vida en adultos mayores del Centro de Salud Los Aquijes, Ica 2019. Material y métodos: Estudio descriptivo, cuantitativo, transversal, diseño correlacional; muestra de estudio con 97 adultos mayores, aplicando la técnica de encuesta y un cuestionario validado por expertos. Resultados: Características sociodemográficas predominantes: Edad 6065 años (52,6\%), sexo femenino (66\%), estado civil casado (37,1\%), instrucción educativa secundaria $(46,4 \%)$. El 30,9\% sufre de hipertensión arterial y $69,1 \%$ no. Los adultos mayores tienen información preventiva global de nivel medio $64,9 \%$, nivel bajo $18,6 \%$, y nivel alto de información con $16,65 \%$. Por dimensiones la información sobre conceptos generales de la hipertensión arterial, información sobre complicaciones, e información sobre cuidado-prevención, es en mayor proporción de nivel medio $(69,1 \%, 50,5 \%$, y $67 \%$ respectivamente). Globalmente los adultos mayores tienen un estilo de vida regular $72,1 \%$, malo $15,5 \%$, y bueno $12,4 \%$. Por dimensiones el estilo de vida es regular en alimentación $60,8 \%$, actividad física $74,2 \%$, consumo de sustancias nocivas $80,4 \%$, y responsabilidad por la salud $79,4 \%$. No existe relación significativa entre la información preventiva y el estilo de vida que practican los adultos mayores del Centro de Salud Los Aquijes, Ica 2019, al encontrarse un valor $p=0,080$ según la prueba de Chi cuadrado $\left(\mathrm{X}^{2}\right)$. Conclusiones: La información preventiva sobre hipertensión arterial es de nivel medio y el estilo de vida es regular. Se acepta la hipótesis nula, al no encontrar relación entre ambas variables.

Palabras clave: Información, hipertensión arterial, estilo vida, adultos mayores.

\section{SUMMARY}

The study aimed to Objective to: Determine the relationship between preventive information on arterial hypertension and lifestyle in older adults of the Los Aquijes Health Center, Ica 2019. Material and methods: Descriptive, quantitative, cross-sectional study, correlational design; Study sample with 97 older adults, applying the survey technique and a questionnaire validated by experts. Results: Predominant sociodemographic characteristics: Age 60-65 years (52.6\%), female sex $(66 \%)$, married marital status $(37.1 \%)$, secondary education instruction $(46.4 \%)$. $30.9 \%$ suffer from high blood pressure and $69.1 \%$ do not. Older adults have a global preventive information of medium level $64.9 \%$, low level $18.6 \%$, and high level of information with $16.65 \%$. By dimensions the information on general concepts of arterial hypertension, information on complications, and information on care and prevention, is in a higher proportion of medium level $(69.1 \%, 50.5 \%$, and $67 \%$ respectively). Globally older adults have a regular lifestyle $72.1 \%$, bad $15.5 \%$, and good $12.4 \%$. By dimensions the lifestyle is regular in food $60.8 \%$, physical activity $74.2 \%$, consumption of harmful substances $80.4 \%$, and health responsibility $79.4 \%$. There is no significant relationship between the preventive information and the lifestyle practiced by older adults at the Los Aquijes Health Center, Ica 2019, when a value $p=0.080$ is found according to the Chi square test (X2). Conclusions: The preventive information on arterial hypertension is of medium level and the lifestyle is regular. The null hypothesis is accepted, finding no relationship between both variables.

Keywords: Information, hypertension, lifestyle, older adults. 


\section{INTRODUCCIÓN}

La hipertensión arterial, es una enfermedad cardiovascular conocida actualmente como "la enfermedad silenciosa", por no presentar síntomas iniciales, y caracteriza por un aumento en los niveles de presión de la sangre, causando un daño cardiovascular, específicamente en las arterias coronarias, constituyendo un gran problema de salud que puede manejarse terapéuticamente con la adopción de estilos de vida saludable y autocuidado de la salud. En la actualidad las enfermedades cardiovasculares cobran una gran proporción de mortalidad en la población, esto como consecuencia de una alimentación inadecuada con excesivo consumo de grasas, falta de actividad física, sedentarismo, entre otros factores que dan origen a la aparición de la enfermedad. En personas adultas mayores que sufren de esta condición, es necesario que mantengan su estado de salud evitando los malos hábitos de vida que se practican, siendo la principal herramienta el "autocuidado" en el paciente, ya que su salud depende de su responsabilidad para con su salud, realizando buenas prácticas o conductas saludables que le permitan contrarrestar las complicaciones de la hipertensión arterial (1).

Según un estudio a nivel internacional, las actitudes hacia la prevención de la hipertensión arterial en adultos mayores son deficientes, lo que ocasiona que no tengan la disposición y deseo por prevenir dicha enfermedad (2). De igual forma un estudio realizado por especialistas en Lima Perú, concluyen que el conocimiento global en adultos mayores con respecto a la hipertensión arterial y su prevención es de nivel medio y bajo, lo que genera la aparición de complicaciones cardiovasculares y la muerte (3).

Ante esta situación, uno de los objetivos de la salud pública, es abordar la problemática del aumento de la hipertensión arterial en la población, haciendo un análisis de la situación de salud en una determinada comunidad con el diagnóstico y tratamiento efectivo de las personas con esta enfermedad, y aplicando actividades preventivo-promocionales con la finalidad de modificar conocimientos o información que poseen los individuos, la familia y comunidad en conjunto, logrando de esta manera que tomen conciencia sobre la importancia del autocuidado de la salud con la adopción de buenos estilos de vida, logrando de esta manera que la enfermedad de la hipertensión arterial aparezca(4).

Considerando todos estos enunciados, se hizo necesario que se aborde la problemática de la deficiente información sobre la hipertensión arterial que posee la población adulta mayor, situación es reportada en la literatura médica como una situación negativa, evidenciándose que muchos adultos mayores que acuden a un centro de salud por algún problema, no tienen información 0 conocimiento necesario para prevenir la enfermedad de la hipertensión arterial, por lo que se hizo necesario ejecutar el presente estudio, el cual permitió conocer la magnitud del problema de la deficiente información en adultos mayores, a la vez analizar si existe una relación directa entre esta variable y la práctica del estilo de vida que realiza este grupo poblacional en riesgo, lo cual permitió que el equipo de salud tenga un mejor conocimiento de dicha problemática, y proponer acciones de salud pública en el marco de la prevención de la enfermedad hipertensiva para una mejor calidad de vida en adultos mayores(5).

Por ello el estudio, se ejecutó en adultos mayores que acuden al Centro de Salud del Distrito de Los Aquijes de la Provincia de Ica en Perú en el año 2019, con el objetivo de analizar la información sobre prevención de la hipertensión arterial, y a su vez determinar el tipo de estilo de vida que llevan dichos usuarios, el cual puede ocasionar que aparezcan problemas cardiovasculares como es el caso de la Hipertensión Arterial. 


\section{MATERIAL Y MÉTODOS.}

El estudio es de tipo descriptivo, cuantitativo y transversal.

La población estuvo conformada por 130 adultos mayores atendidos en promedio durante un mes, el tamaño de la muestra resultando un total de 97 adultos mayores mediante fórmula estadística finita. La técnica utilizada para la ejecución del estudio fue la encuesta y el instrumento utilizado fue el cuestionario elaborado por la propia investigadora tomando como referencia los instrumentos de los estudios de Tafur C, Vásquez L y Nonaka M. (2017)(6), y Santa Cruz E y Sobrado C. (2016)(7) sujeto a confiabilidad aplicando una prueba piloto obteniéndose una confiabilidad de 0,81 para cuestionario de información preventiva sobre hipertensión arterial y 0,79 para cuestionario estilo de vida, el cuestionario consta de datos sociodemográficos con 5 ítems que comprende edad, sexo, estado civil, instrucción educativa y si padece de hipertensión arterial. La variable información preventiva sobre hipertensión arterial comprende 15 ítems en sus 3 dimensiones y la variable estilo de vida comprende 15 ítems en sus 4 dimensiones.

En la variable información preventiva sobre hipertensión arterial se utilizó un formulario de tipo escala vigesimal con una medición de nivel alta información, regular información y baja información, la variable estilo de vida se realizó utilizando la escala de Likert con los índices (Siempre, A veces, Nunca) con nivel de medición de Buen estilo de vida, regular estilo de vida y mal estilo de vida. Para el procesamiento de datos se utilizó la estadística descriptiva, el uso de Excel 2019 y SPSS Versión última 25.

Para la contrastación Se utilizó prueba estadística de chi cuadrado $\left(\mathrm{X}^{2}\right)$ de análisis bivariado con significancia de $\mathrm{p}<0.05 \mathrm{y}$ confiabilidad de $95 \%$, obteniéndose un valor de 8,34 aceptándose la $\mathrm{H}_{0}$.

\section{RESULTADOS}

En relación a la edad de los adultos mayores del Centro de Salud Los Aquijes predominan las edades de 60 a 65 años con 52,6\% (51 adultos mayores), en cuánto al sexo hay mayor porcentaje de mujeres con 66\% (64 adultos mayores), en cuánto al estado civil predomina los adultos mayores casados con $37,1 \%$ (36 adultos mayores), en el nivel educativo predominan los adultos mayores con educación secundaria con 46,4\% (adultos mayores), en cuánto al diagnóstico de la HTA un $30,9 \%$ (30 adultos mayores) lo padece y $69,1 \%$ (67 adultos mayores) no lo padece. En la tabla 1 se analizó la información preventiva sobre hipertensión arterial dando como resultado nivel medio $64,9 \%$, seguido de la baja información con $18,6 \%$ y alta información con $16,5 \%$. En el Gráfico 1 se analizó la información preventiva sobre hipertensión arterial sobre la Dimensión Conceptos Generales obteniéndose como resultado, mediana información con $69,1 \%$, seguido de la baja información con $17,5 \%$ y de la alta información con 13,4\%. En la información sobre Complicaciones se obtuvo como resultado, media información con 50,5\%, seguido de la baja información 35,1\% y alta información con $14,4 \%$. Por último, se analizó la información sobre CuidadoPrevención obteniéndose como resultado, media información con $67 \%$, seguido de la alta información con $22,7 \%$ y baja información con 10,3\%. En la Tabla 2 se analizó el estilo de vida de los adultos mayores dando como resultado, presentan regular estilo de vida con $72,1 \%$, seguido del mal estilo de vida con 15,5 y del buen estilo de vida con $12,4 \%$. Se analizó el estilo de vida sobre la dimensión alimentación encontrándose, con regular alimentación $60,8 \%$, seguido de la buena alimentación con $23,7 \%$ y la mala alimentación con $15,5 \%$. En el Gráfico 2 se analizó el estilo de vida sobre la Dimensión: Actividad Física encontrándose como resultado, regular actividad física con $74,2 \%$, seguido de la mala actividad física con $17,5 \%$, y buena 
actividad física con 8,2\%. Se analizó el estilo de vida sobre la Dimensión: Conductas Nocivas encontrándose como resultado, regular conducta nociva con $80,4 \%$, seguido del $10,3 \%$ no lo practican (bueno), y $9,3 \%$ lo realizan frecuentemente conductas nocivas (malo). Se analizó el estilo de vida sobre la dimensión responsabilidad por su salud, encontrándose como resultado, regular responsabilidad por su salud con $79,4 \%$, seguido de la buena responsabilidad por su salud con $13,4 \%$ y la mala responsabilidad por su salud con $7,2 \%$.

Tabla 1. Información Preventiva Sobre Hipertensión Arterial en adultos mayores, Centro de Salud Los Aquijes, Ica 2019

\begin{tabular}{|lcc|}
\hline $\begin{array}{c}\text { Información preventiva } \\
\text { sobre Hipertensión arterial: } \\
\text { Global }\end{array}$ & $\mathbf{N}^{\circ}$ & $\%$ \\
\hline $\begin{array}{l}\text { Alta información } \\
\text { Media información }\end{array}$ & 16 & 16,5 \\
Baja información & 63 & 64,9 \\
\hline & 18 & 18,6 \\
\hline
\end{tabular}

Fuente: Base De Datos

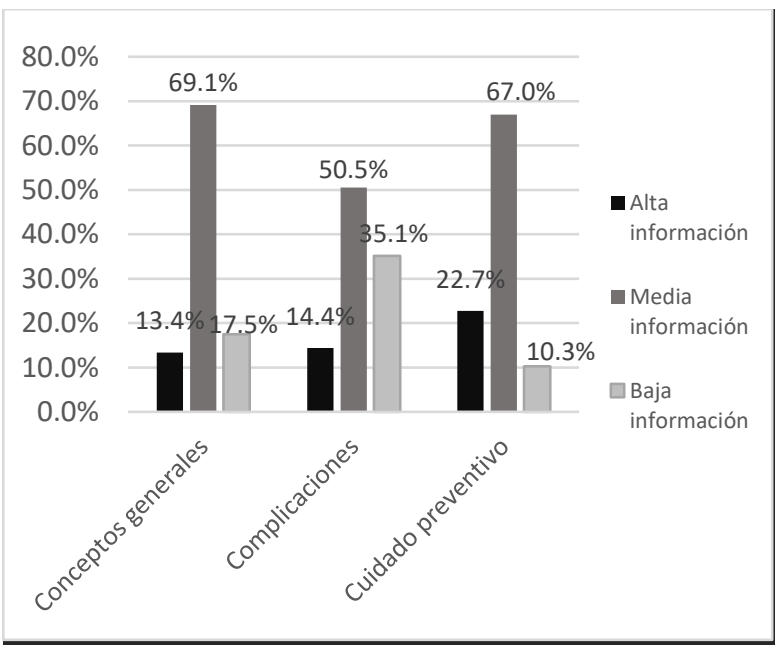

Gráfico 1. Información Preventiva Sobre Hipertensión Arterial según dimensiones, Centro de Salud Los Aquijes, Ica 2019

Tabla 2. Estilo de vida en Adultos Mayores, Centro de Salud Los Aquijes, Ica 2019

\begin{tabular}{|c|c|c|}
\hline Estilo de Vida: Global & $\mathbf{N}^{\circ}$ & $\%$ \\
\hline Buen estilo de vida & 12 & 12,4 \\
\hline Regular estilo de vida & 70 & 72,1 \\
\hline Mal estilo de vida & 15 & 15,5 \\
\hline Total & 97 & $100 \%$ \\
\hline
\end{tabular}

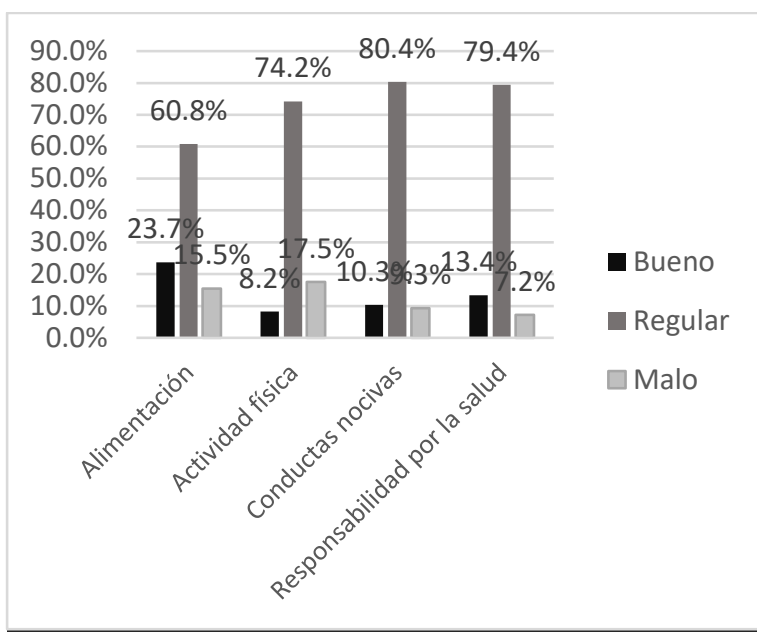

Gráfico 2. Estilo de Vida en Adultos Mayores según dimensiones, Centro de Salud Los Aquijes, Ica 2019

\section{DISCUSIÓN.}

El estudio tuvo como objetivo general determinar si existía relación entre la información preventiva que poseían los adultos mayores sobre la hipertensión arterial con el estilo de vida que practicaban, quienes acudían al Centro de Salud de Los Aquijes en el año 2019.

Al analizar el nivel de información sobre hipertensión arterial en los adultos mayores del Centro de Salud Los Aquijes, Ica 2019, se encontró en forma global, que es de nivel medio con $64,9 \%$, nivel bajo $18,6 \%$, y nivel alto de información con $16,65 \%$, es decir los adultos mayores no tienen toda la información necesaria sobre la enfermedad, y al encontrar un gran porcentaje de medio y baja información, condicionaría a que no practiquen buen estilo de vida para prevenir la enfermedad. Estos resultados concuerdan con el estudio de Rivera A, Orozco B, Gómez C, y Suarez M. (2015) (2), quienes encontraron en sus resultados que el conocimiento sobre dicha enfermedad era mayormente de nivel regular con un $71,86 \%$ $y$ alto $28,14 \%$. Asimismo, el estudio de Barraza L, y Sarmiento C. (2014) (8) encontraron conocimiento de nivel deficiente en un $70 \%$ en adultos mayores. El estudio a nivel nacional de Carnero B. (2018) (3) ejecutado en Lima, evidenció también resultados similares, al encontrar un $50 \%$ de 
adultos mayores que tenían conocimiento de nivel medio sobre la enfermedad, y el estudio de Tafur C, Vásquez L, y Nonaka M. (2017) (6) encontró resultados similares, al evidenciar un $79 \%$ con conocimiento 0 información inadecuado o deficiente sobre la hipertensión arterial. Autores como Coaquira T, y Tello L. (2015) (9) también reportan que el conocimiento sobre dicha enfermedad en adultos mayores es de nivel medio $y$ deficiente en un $60 \%$. Sin embargo, nuestros resultados difieren con el estudio de Santa Cruz E, Sinche A, y Sobrado C. (2016) (7), quien encontró en sus resultados que el conocimiento sobre hipertensión arterial en adultos mayores es de nivel alto en un $50 \%$.

El nivel de información también fue evaluado en el estudio, según dimensiones, evidenciándose los siguientes resultados: Dimensión Conceptos generales de la Hipertensión Arterial: El 69,1\% de los adultos mayores del Centro de Salud Los Aquijes tienen información de nivel medio sobre los conceptos generales de la hipertensión arterial, 17,5\% tiene información de nivel bajo, y 13,4\% nivel alto de información. Dimensión: Complicaciones de la Hipertensión Arterial: El 50,5\% de los adultos mayores del Centro de Salud Los Aquijes tienen información de nivel medio sobre las complicaciones de la hipertensión arterial, $35,1 \%$ tiene información de nivel bajo, y $14,4 \%$ nivel alto de información sobre las complicaciones de la hipertensión arterial. Dimensión: Cuidado - prevención de la hipertensión arterial: El 67\% de los adultos mayores del Centro de Salud Los Aquijes tienen información de nivel medio sobre el cuidado de la salud para la hipertensión arterial, 22,7\% tiene información de nivel alta, y 10,3\% nivel bajo de información sobre el cuidado de la salud en la hipertensión arterial. Estos resultados concuerdan con Barraza L, y Sarmiento C. (2014) (8), quien encontró en sus resultados que la mayoría de los adultos mayores tiene niveles medios y bajos sobre los conceptos de la enfermedad tales como la sintomatología y conocimiento sobre información en un 55\%.
Asimismo, el estudio de Carnero B. (2018) (3) evidencia que las dimensiones de información en cuidado - prevención de la hipertensión arterial son mayormente de nivel medio con $45 \%$, relacionados a la alimentación que deben realizar y conocimiento sobre la actividad física de nivel medio con un $52 \%$.

Al analizar el estilo de vida que practicaban los adultos mayores, se evidencia en forma global que $72,1 \%$ de los adultos mayores del Centro de Salud Los Aquijes Ica 2019 poseen un regular estilo de vida, $15,5 \%$ mal estilo de vida, y $12,4 \%$ poseen un buen estilo de vida, resultados que difieren con el estudio de Carbajal A, y Huamanculí F. (2018) (10) quienes encontraron en su estudio que el estilo de vida mayormente es medio o no saludable con un $42,5 \%$ y $57,5 \%$ saludable. El estudio de Carbajal L, y Sayas E. (2018) (11) coincide con el estudio al reportar en sus resultados que el estilo de vida en relación a la práctica de autocuidado es regular, deficiente o inadecuado en un $80 \%$ y adecuado $20 \%$. Sin embargo, nuestros resultados difieren con el estudio de Burbano D. (2017) (12), quien encontró en sus resultados que el estilo de vida que realizaban los adultos mayores hipertensos era mayormente bueno en un $70 \%$.

El estilo de vida se analizó por dimensiones, encontrando los siguientes resultados: $60,8 \%$ de los adultos mayores del Centro de Salud Los Aquijes de Ica practican una regular alimentación, 23,7\% tiene una buena alimentación, y 15,5\% realizan una mala alimentación. El $74,2 \%$ de los adultos mayores del Centro de Salud Los Aquijes de Ica practican una regular actividad física, $17,5 \%$ tienen una mala actividad física, y $8,2 \%$ realizan una buena actividad física. El $80,4 \%$ de los adultos mayores del Centro de Salud Los Aquijes de Ica practican regularmente conductas nocivas como el consumir alcohol o tabaco, $10,3 \%$ no lo practican, y $9,3 \%$ realizan frecuentemente conductas nocivas. El $79,4 \%$ de los adultos mayores del Centro de Salud Los Aquijes de 
Ica practican un regular cuidado de la salud tales como el relajarse, acudir al centro de salud o leer revistas para mejorar su estado de salud, $13,4 \%$ tienen buena responsabilidad, y $7,2 \%$ tienen mala responsabilidad por la salud.

Las características sociodemográficas más predominantes en los adultos mayores son: Edad $60-65$ años (52,6\%), sexo femenino (66\%), estado civil casado (37,1\%), instrucción educativa secundaria (46,4\%). Se observa asimismo que $30,9 \%$ sufre de hipertensión arterial y 69,1\% no.

\section{CONCLUSIONES.}

1. Los adultos mayores tienen una información preventiva global sobre hipertensión arterial de nivel medio $64,9 \%$, nivel bajo $18,6 \%$, y nivel alto de información con 16,65\%. Por dimensiones la información es de nivel medio en las 3 dimensiones: Conceptos generales de la hipertensión arterial $69,1 \%$, información sobre complicaciones $(50,5 \%)$, e información sobre cuidadoprevención de la hipertensión arterial (67\%).

2. El estilo de vida de los adultos mayores es regular $72,1 \%$, malo $15,5 \%$, bueno $12,4 \%$. Por dimensiones el estilo de vida es mayormente regular en la alimentación $60,8 \%$, actividad física $74,2 \%$, consumo de sustancias nocivas $80,4 \%$, responsabilidad por la salud con $79,4 \%$.

3. No existe relación significativa entre la información preventiva sobre hipertensión arterial y el estilo de vida que practican los adultos mayores del Centro de Salud Los Aquijes, Ica 2019, al encontrarse un valor $\mathrm{p}=0.080$ según la prueba de Chi cuadrado (X2).

\section{CORRESPONDENCIA}

Lic. Enf. Fátima Gregoria Huamán Vega.

Correo Eléctrico:

Fata_18_4@hotmail.com

\section{REFERENCIAS BIBLIOGRÁFICAS.}

1. Patel $P$, Ordunez $P$, DiPette D, Escobar M, Hassel T, Wyss F, et al. Control de la presión arterial para reducir la morbilidad y mortalidad por enfermedades cardiovasculares: Proyecto de prevención y tratamiento. Rev. Panam. Salud Pública 2017; 41(8).

2. Rivera A, Orozco B, Gómez C, Suarez M. Conocimiento, actitudes y prácticas del paciente hipertensión del Hospital Malvinas, Florencia. Tesis de grado. Huila, Colombia. Universidad Surcolombiana-Ciencias de la Salud; 2015

3. Carnero B. Nivel de conocimiento en adultos mayores sobre prevención y complicaciones de Hipertensión Arterial en Geriatría del hospital Dos de Mayo. Tesis de grado. Lima, Perú. Univ. Privada San Juan Bautista; 2018

4. Campos I, Hernández L, Pedroza A, Medina C, Barquera S. Hipertensión arterial en adultos mexicanos. Rev. Salud Pública de México 2018; 60(3)

5. Instituto Nacional de Salud Pública. Prevenir la Hipertensión arterial. (Internet) México, 2017. (Citado el 16 Agosto 2019). Disponible en: https://www.insp.mx/avisos/3646hipertension-arterial.html.

6. Tafur C, Vásquez L, Nonaka M. Nivel de conocimiento y práctica de autocuidado en adulto mayor hipertenso en Hospital Regional de Loreto, Iquitos. Tesis de grado. lquitos, Perú. Universidad Nacional de la Amazonía Peruana; 2017.

7. Santa Cruz E, Sinche A, Sobrado C. Conocimiento y prácticas de autocuidado en adultos mayores hipertensos en 3 establecimientos de salud de Huánuco. Tesis de grado. Huánuco, Perú. Facultad de Enfermería, Universidad Nacional Hermilio Valdizán; 2016. 
8. Barraza L, Sarmiento C. Conocimiento de la enfermedad hipertensión en una población colombiana. Rev. De la Facultad de Medicina. Colombia, 2014; 62(3): 399-405.

9. Coaquira T, Tello L. Nivel de conocimiento de Hipertensión Arterial en adultos del Servicio medicina del Hospital Huaycán. Tesis de grado. Lima, Perú Facultad de Enfermería, Universidad Norbert Wiener; 2015.

10. Carbajal A, Huamanculí F. Estilo de vida del adulto mayor en el Centro de Salud la Palma, Ica. Tesis de grado. Ica, Perú. Universidad Nacional San Luis Gonzaga Ica; 2018.
11. Carbajal L, Sayas E. Nivel de conocimiento y práctica de autocuidado en adulto mayor hipertenso, Hospital Sergio Bernales de Collique, Comas. Tesis de grado. Lima, Perú. Lima, Facultad de Enfermería, Universidad Peruana Los Andes; 2018.

12. Burbano D. Estilo de vida y calidad de vida en salud de usuarios hipertensos. [tesis de posgrado] Universidad Nacional de Colombia-Enfermería. Bogotá, Colombia; 2017.

Recibido: 03/02/2021

Aprobado para publicación: 07/05/2021 